\title{
DE POTRON-MINET À ENTRE CHIEN ET LOUP... QUE DE CHATS !
}

\author{
Jean-Pierre GoudAILLIER \\ Université Paris Descartes
}

\begin{abstract}
The French language contains a large number of phrases, expressions with the lexeme cat. The cat has been the favorite domestic animal of the French for a very long time and many authors of French francophone literature offer us, among others, aphorisms and maxims and give an important place to cat(s) in their writings. Many sayings refer to this animal. In the limited context of this text it is impossible to make an exhaustive survey of all the cat occurrences contained in the language, but the various examples that are selected and presented allow having an idea of the symbols, to which the cat is associated, and emotions that it arouses.
\end{abstract}

Ce petit texte "félino-linguistique » à l'adresse d'une grande amoureuse des chat(te)s de la part d'un non moins amoureux des félins de tous poils. Bon anniversaire.

«Que vous soyez fier comme un coq

Fort comme un boeuf

Têtu comme un âne Malin comme un singe Ou simplement un chaud lapin Vous êtes tous, un jour ou l'autre Devenu chèvre pour une caille aux yeux de biche Vous arrivez à votre premier rendez-vous

Fier comme un paon

Et frais comme un gardon

Et là... Pas un chat ! Vous faites le pied de grue 
Vous demandant si cette bécasse Vous a réellement posé un lapin

Il y a anguille sous roche... »

Jean d'Ormesson ${ }^{1}$

L'aube, la pointe du jour, le petit matin appartient, du moins appartiendrait aux chats, s'il on en croit son ancienne appellation datant de 1835, désormais désuète, potron-minet. Au crépuscule, entre chien et loup, les canins closent la période diurne. La langue française, comme toute autre langue, fourmille d'expressions, de locutions, de dictons, etc. qui font appel aux chiens, mais surtout aux chats.

Potron-minet, parfois déformé en patron-minet ou poltron-minet, est essentiellement utilisé dans les locutions à potron-minet, dès potron-minet, dès le potron-minet :

[...] il m'a même engueulé [...] je lui ai juré sur tout ce que j'avais de plus sacré que, dès que vous seriez revenu dans votre bureau, je vous ferais un rapport complet; et que de votre côté vous ne manqueriez pas dès potronminet d'exposer au général Joffre toute la gravité de la situation. (Romains, $1938: 194)$

[...] les trains étaient déjà tous partis ou arrivés, et les cheminots n'en attendaient plus d'autres avant potron-minet. (Queneau, 1942 : 19)

[...] cependant qu'une rafale de mistral par terre et sur les toits faisait s'envoler les feuilles mortes des platanes centenaires du cours Mirabeau, les branches qui s'entrechoquaient couvrant la voix chevrotante du vieil homme chinois et que la fumée de ma cheminée qui refluait dans la chambre me brûlait les yeux, réveillant en moi des lointaines réminiscences de calorifère allumé à potron-minet, de cave, de poux, de misère, de faim, de haillons [...]. (Cendrars, $1948: 353$ )

Georges Brassens dans la chanson « Le revenant» emploie non pas à potronminet mais au potron-minet :

Il la trouve se réchauffant

Avec un salaud de vivant,

Alors chancelant dans sa foi

\footnotetext{
${ }^{1}$ https://ecumedesmots.wordpress.com/2017/01/05/le-francais-une-langue-animale-jean-dorm esson (dernière consultation : le 06.12.2017).
} 
Mourut une seconde fois.

La commère au potron-minet

Ramassa les os qui traînaient

Et pour une bouchée de pain

Les vendit à des carabins.

(Brassens, 1973 : 378)

D'après le TLFi, patron-minet, autre forme de potron-minet, est parfois altéré en patron-jaquet, patron-jacquette, voire patron-minette ; certaines de ces variantes auraient été utilisées, toujours selon le TLFi, par Honoré de Balzac dans Le père Goriot $(1835: 50)$ et Victor Hugo dans le Tome 1 de son œuvre fleuve Les Misérables (1862: 862). Par ailleurs, le TLFi précise que dès potron-minet est une locution adverbiale formée par substitution de minet (chat) à jacquet (écureuil). Potron, poitron à la fin du $\mathrm{XII}^{\mathrm{e}}$ siècle représentent

[...] le b. lat. posterio « cul ». Cette loc. qui signifie proprement « dès que le derrière de l'écureuil se fait voir ", s'explique par le fait que l'écureuil dresse souvent sa queue, faisant ainsi voir son derrière. Son remplacement par potron-minet est sans doute dû au fait que le chat passe pour être très matinal ${ }^{2}$.

Toujours à propos des chats, le $T L F i$, à l'entrée chat, rappelle l'existence de l'adverbe chattement considéré comme familier, qui signifie 'à la manière douce et enjôleuse des chattes' : « Elle alla chattement à lui » (Balzac, $1848: 129)^{3}$.

Les chiens n'en sont pas pour autant oubliés par la langue française et à l'entrée chien du $T L F i$ on relève entre chien et loup, qui dans la langue populaire renvoie au moment du crépuscule, auquel on ne fait pas la distinction entre un chien et un loup, car tous les objets se confondent à cette heure :

[...] Un bon coup de tromblon dans les fesses ! Nos pionniers, ils se montraient plus... Ils sortaient seulement avant l'aube, juste à peine une heure ou deux entre chien et loup... au tout petit matin pour y voir quand même un peu clair [...]. (Céline, 1936 : 1027)

Louis-Ferdinand Céline dans cet exemple semble faire un usage erroné d'entre chien et loup, puisqu'il est question de l'aube et non du crépuscule.

\footnotetext{
${ }^{2} T L F i$.

${ }^{3} T L F i$.
} 
Entre chien et loup est attesté dès le XVII ${ }^{\mathrm{e}}$ siècle :

[...] et les profanations que les libertins de nostre temps inventent journellement seroient peut-estre mieux au centre de la terre, que dans des livres imprimez, mais puis qu'elles voltigent comme chauve-souris entre chien et loup, et parmy des esprits qui ne sont ny bons catholiques ny mauvais hérétiques, mais flottant entre les deux, je suis d'advis de les tirer en plein jour pour faire voir leur difformité, et les exposer à la rizée de tout le monde. (Garasse, 1623 : 198, Livre 2, Section 15)

Quoique je ne m'inquiète de rien, je me vois servie par de petits ordres invisibles. Je me promène seule, mais je n'ose me livrer à l'entre chien et loup, de peur d'éclater en cris et en pleurs. L'obscurité me serait mauvaise dans l'état où je suis ; si mon âme peut se fortifier, ce sera à la crainte de vous fâcher que je sacrifierai ce triste divertissement. (Sévigné (de), 1696 : 143) ${ }^{4}$

La littérature des $\mathrm{XIX}^{\mathrm{e}}$ et $\mathrm{XX}^{\mathrm{e}}$ siècles offre de nombreux exemples d'utilisation d'entre chien et loup, parmi lesquels on peut relever :

On dîne en plein air, les amis viennent tantôt l'un, tantôt l'autre, on fume, on jase, et le soir quand ils sont partis Chopin me joue du piano entre chien et loup après quoi il s'endort comme un enfant en même temps que Maurice et Solange. Moi je lis l'Encyclopédie et je prépare ma leçon du lendemain. (Sand, $1839: 684)^{5}$

Alphonse Boudard, quant à lui, n' hésite pas à utiliser cette expression populaire dans un contexte argotique comptant pas moins de six lexèmes de ce registre en quatre phrases courtes :

Même aux brèmes j'ai rarement beau schpile, j'ose plus les toucher, je m'écarte des tripots. Rien à chiquer, je suis pris. C'est ça la Cerise, l'existence entre chien et loup, entre deux douleurs, entre deux gendarmes. Pour plus de détails, tournez la page. Soyez sans crainte, je préfère tout de même vous faire marrer, que vous en ayez pour votre pognon. (Boudard, $1963: 15)^{6}$

${ }^{4}$ Source: Frantext.

${ }^{5}$ Source: Frantext.

${ }^{6}$ Alphonse Boudard, dans un autre texte, utilise lui aussi dès potron-minet : « La richesse c'est un ensemble, ça commence dès potron-minet... on pose pas ses panards sur les mêmes tapis... on pète pas dans les mêmes calcifs... on lève pas le petit doigt pour les mêmes raisons... xétera » (Boudard, 1995 : 146). 
Winston Churchill ${ }^{7}$ tend une passerelle entre l'univers canin et celui des félins, mais il avance un point de vue bien arrêté au sujet des chats et des chiens, car sans ambages il fait part de son observation : «Les chiens vous regardent tous avec vénération. Les chats vous toisent tous avec dédain » (Churchill, Enright, 2014 : 54) avant de préciser : «Il n’y a que les cochons qui vous considèrent comme leurs égaux $»$ (ibid.).

Les chats ont de tous temps inspiré grand nombre d'auteur(e)s français(e)s et francophones ${ }^{8}$ qui nous offrent, entre autres, une multitude d'aphorismes, voire des maximes. En tout bien tout honneur on peut donner la parole à SidonieGabrielle Colette, grande amoureuse des chats ${ }^{9}$. Pour elle « le temps passé avec un chat n'est jamais perdu » et «à fréquenter les chats, on ne risque que de s'enrichir », car « Il n'y a pas de chat ordinaire $»^{10}$.

Guy de Maupassant n'est pas en reste ; pour lui « les chats c'est comme le papier, ça se froisse très vite » et « rien n'est plus doux, rien ne donne à la peau une sensation plus délicate, plus raffinée, plus rare que la robe tiède et vibrante d'un chat $»^{11}$.

Stéphane Mallarmé constate que "Les chats sont des êtres faits pour emmagasiner la caresse $»^{12}$.

Prenons garde cependant. À en croire Théophile Gautier, « Si vous êtes digne de son affection, un chat deviendra votre ami mais jamais votre esclave $»^{13}$.

Jean Cocteau nous rappelle que « Petit à petit les chats deviennent l'âme de la maison » et nous dit avec une certaine pointe d'humour : «J'aime les chats car il n'existe pas de chat policier $\gg^{14}$.

Le chat inspire à Eugène Ionesco un syllogisme : « Tous les chats sont mortels, Socrate est mortel, donc Socrate est un chat $\rangle^{15}$. Pourquoi pas !

${ }^{7}$ Rab Butler, chancelier de l'Échiquier, a lui-même raconté que lors d'une visite, en 1943, il avait trouvé Churchill au lit et Nelson (le chat de Churchill) roulé en boule sur ses pieds : « Ce chat, lui avait déclaré le Premier ministre, contribue plus que vous à l'effort de guerre : il me sert de bouillotte, et me fait ainsi économiser du chauffage » (Churchill, Enright, $2014: 60$ ).

${ }^{8}$ Ils inspirent aussi les peintres, qui ne se contentent pas de les reproduire dans leurs tableaux mais peuvent aussi à l'occasion écrire à leur sujet, tout comme Alberto Giacometti qui précise : « dans un incendie, entre un Rembrandt et un chat, je sauverais le chat » (http://www.micetto.com/ citations-sur-les-chats [dernière consultation : le 07.12.2017]).

${ }^{9}$ D'après Frantext, Sidonie-Gabrielle Colette utilise 355 fois le lexème chat dans son œuvre.

${ }^{10}$ Source : $\mathrm{http}: / /$ www.micetto.com/citations-sur-les-chats (dernière consultation : le 07.12.2017).

${ }^{11}$ Ibid.

${ }^{12}$ Ibid.

${ }^{13} \mathrm{Ibid}$.

${ }^{14}$ Source: https://www.absolumentchats.com/60-citations-sur-les-chats (dernière consultation : le 05.12.2017).

${ }^{15}$ Source : http://www.micetto.com/citations-sur-les-chats (dernière consultation : le 07.12.2017). 
Les proverbes relatifs aux chats sont très nombreux en français, ce qui est aussi le cas de beaucoup d'expressions. Ainsi, le fait de ne pas distinguer certains détails dans l'obscurité est exprimé par La nuit tous les chats sont gris. Des chiens ne font pas des chats rappelle que l'on conserve toutes et tous une hérédité, les caractéristiques de nos parents d'un point de vue biologique. L'expression très connue Quand le chat n'est pas là, les souris dansent signifie que lorsque le supérieur hiérarchique est absent, ses subalternes en profitent pour faire ce que bon leur semble. La traduction de l'expression correspondante en italien est Quand le chat court sur les toits, les souris dansent au logis. Aux vilains matous les belles chattes renvoie au fait que les jolies filles sont bien souvent attirées par les mauvais garçons. Lorsque l'on a la chance d'avoir une très bonne vision, surtout la nuit, dans l'obscurité, on se vante d'avoir des yeux de chat. Avoir un ceil qui surveille le poisson et l'autre qui fait attention au chat est une expression populaire « piednoire », que l'on trouve par conséquent en pataouète ${ }^{16}$ pour désigner un strabisme très divergent. Lorsque l'on a un chat dans la gorge, on est enroué et si l'on donne sa langue au chat, on reconnaît ne pas savoir la réponse à une question posée et l'on avoue ainsi son ignorance. Se laver sommairement revient à faire une toilette de chat. Si l'on a une grande mésentente avec une personne, que l'on entretient de ce fait avec celle-ci des rapports houleux, s'entendre comme chien et chat s'applique à un tel cas. En employant il n'y a pas de quoi fouetter un chat, on veut dire que la chose, l'affaire en question n'a guère d'importance. Par contre avoir d'autres chats à fouetter a pour sens avoir des choses plus importantes à faire. Lorsqu'il n'y a pas un chat, c'est qu'il n'y a personne, vraiment personne ${ }^{17}$. Avoir une vie de chat, c'est avoir une vie facile, confortable, contrairement à avoir une vie de chien qui signifie que l'on a une vie difficile, pénible, voire misérable. Appeler un chat un chat, c'est dire les choses sans détour, telles qu'elles sont. Retomber comme un chat sur ses pattes signifie que l'on s'en sort toujours, même lorsque l'on se trouve dans une situation délicate. Si une boisson, plus particulièrement une bière, n'a aucune saveur, aucun goût, c'est du pipi de chat et si un travail est bâclé, c'est de la bouillie pour les chats. On le dit aussi d'un texte incompréhensible. Écrire comme un chat, c'est avoir une écriture illisible. Pourquoi comme un chat? En

\footnotetext{
${ }^{16}$ Le pataouète est le parler des quartiers populaires d'Alger au moment de la colonisation. Pour Françoise Gadet et Ralph Ludwig, il comporte « des emprunts aux différentes langues présentes en Algérie à partir du XIX ${ }^{\mathrm{e}}$ siècle, avant tout l'arabe [...] il a aussi connu d'autres apports linguistiques, d'Italie, d'Espagne ou de Malte, mais aussi du provençal, la plupart des colons d'Algérie étant originaires du sud de la France » (Gadet, Ludwig, 2014 : 87). Pour diverses informations au sujet du pataouète on peut se reporter à l'ouvrage de Roland Bacri (1969).

${ }^{17} \mathrm{Cf}$. à ce sujet la citation de Jean d'Ormesson en tête de ce texte. Dans la correspondance de Voltaire on trouve la forme plus ancienne de cette expression : il n'y a pas la queue d'un chat.
} 
argot, un greffier est un chat. On trouve ce lexème dans le Glossaire argotique des mots employés au bagne de Brest d'Ansiaume en 1821. Albert Simonin l'utilise : «J'ai seulement rencontré un greffier, un beau chat noir qui s'est tiré à mon approche » (Simonin, $1953: 136$ ). Dès lors pour quelle raison dit-on écrire comme un chat, comme un greffier donc, pour mal écrire ? Le rapprochement de chat et greffier daterait de 1800 pour Jacques Cellard et Alain Rey, qui pensent que celui-ci est peut-être même antérieur à cette date : " le voisinage greffe (de justice) / griffe (du chat), le premier du latin, le second du francique, mais tous deux reliés par le sens commun "égratignure" (cf. griffe au sens de paraphe), a conduit de bonne heure à l'image du chat justicier (le "greffier") » (Cellard et Rey, $1980: 424$ ).

Dans son poème Les chats, Charles Baudelaire nous fait partager sa propre vision des chats :

Les amoureux fervents et les savants austères

Aiment également, dans leur mûre saison,

Les chats puissants et doux, orgueil de la maison,

Qui comme eux sont frileux et comme eux sédentaires.

(Baudelaire, $1857: 63$ )

Pour finir, on peut citer aussi un extrait du roman beaucoup plus récent, contemporain même, Plume de Guy Féquant :

[...] je réussis à attirer Plume jusque sous le tilleul. Je la pris dans mes bras. Son ronron un peu sec charmait mon oreille. Elle fit pour la première fois quelque chose qu'elle allait renouveler souvent : escalader mon épaule droite et s'allonger de tout son long à l'arrière de mon cou. (Féquant, $2014: 24$ )

En guise de conclusion il s'agit de réaffirmer qu'il existe en français de nombreuses locutions et expressions contenant le lexème chat et de constater que celui-ci est abondamment utilisé dans la littérature française et francophone tant par le passé qu'à l'époque actuelle. Le présent texte ne contient qu'un nombre restreint d'exemples, tous issus d'un important corpus. En effet, que de chats dans la langue et la littérature française ! Et cela du matin au soir, de potron-minet à entre chien et loup. 


\section{Références bibliographiques}

\section{- linguistiques}

ANSIAUME, Louis (1821), Glossaire argotique des mots employés au bagne de Brest-Argot en usage au bagne de Brest et connu des voleurs de toutes les provinces françaises, manuscrit de la main d'un juge de Chartres, Bibliothèque municipale de Rouen (fonds Montbret).

BACRI, Roland (1969), Le roro. Dictionnaire pataouète de la langue pied-noir, Paris, Denoël.

CELLARD, Jacques, REY, Alain (1980), Dictionnaire du français non conventionnel, Paris, Hachette.

GADET, Françoise, LUDWIG, Ralph (2014), Le français au contact d'autres langues, Paris, Ophrys.

Trésor de la Langue Française informatisé (TLFi), Nancy, CNRS, ATILF (Analyse et traitement informatique de la langue française), UMR CNRS-Université Nancy 2.

\section{- littéraires}

BALZAC (de), Honoré (1835), Le père Goriot, Paris, Werdet.

BALZAC (de), Honoré (1848), Le Cousin Pons, Paris, Pétion.

BAUDELAIRE, Charles (1857), Les Fleurs du Mal, Paris, Édition Le Dantec.

BOUDARD, Alphonse (1963), La Cerise, Paris, Plon.

BOUDARD, Alphonse (1995), Mourir d'enfance, Paris, Robert Laffont.

BRASSENS, Georges (1973), Poèmes et chansons, Paris, Seuil.

CÉLINE, Louis Ferdinand (1936), Mort à crédit, Paris, Denoël et Steele.

CENDRARS, Blaise (1948), Bourlinguer, Paris, Denoël.

CHURCHILL, Winston, ENRIGHT Dominique (2014), Les sautes d'humour de Winston Churchill, Paris, Éditions Payot.

FÉQUANT, Guy (2014), Plume, Bouvellemont, Éditions Noires Terres.

GARASSE, François (1623), La Doctrine curieuse des beaux esprits de ce temps, ou prétendus tels,

Paris, S. Chappelet.

HUGO, Victor (1862), Les Misérables, t. 1, Paris, Pagnerre.

QUENEAU, Raymond (1942), Pierrot, Paris, Gallimard.

ROMAINS, Jules (1938), Les Hommes de bonne volonté, t. 16 (Verdun), Paris, Flammarion.

SAND, George (1839), Correspondance, Frantext.

SÉVIGNÉ (de), Madame (1696), Correspondance, t. 3 (1680-1696), Frantext.

SIMONIN, Albert (1953), Touchez pas au grisbi, Paris, Éditions de la Nouvelle Revue Française.

\section{Sitographie}

www.micetto.com/citations-sur-les-chats.

www.ecumedesmots.wordpress.com/2017/01/05/le-francais-une-langue-animale-jean-dormesson.

www.absolumentchats.com/60-citations-sur-les-chats. 